\title{
NOVO ENCONTRO DO TRYPANOSOMA (MEGATRYPANUM) FREITASI, PARASITA DO GAMBÁ
}

\author{
Eduardo Olavo da Rocha e Silva* \\ Dino Baptista Germano Pattoli ** \\ José de Campos Camargo ***
}

RSPU-B/305

\begin{abstract}
Rocha E Silva, E. O. et al. - Novo encontro do Trypanosoma (Megatrypanum) freitasi, parasita do gambá. Rev. Saúde públ., S. Paulo, 10:121-4, 1976.

RESUMo: Relata-se um novo encontro do Trypanosoma (Megatrypanum) freitasi, raro tripanosomatideo encontrado no sangue de marsupiais, do gênero Didelphis. Salientam-se a baixa e irregular parasitemia observada, as dificuldades no isolamento, bem como, o concomitante achado do T. cruzi.
\end{abstract}

Unitermos: Tripanosomatideos. Tripanossomose americana. Reservatórios.

\section{INTRODUCAO}

Rego et al. ${ }^{2}$ em 1957, descreveram com detalhes o encontro nos terrenos da Faculdade de Medicina de Ribeirāo Preto, antiga Fazenda Monte Alegre, de dois exemplares do Didelphis paraguagensis $(=D$. azarae $)$ parasitados por formas sanguícolas de um tripanosomatídeo até então desconhecido, ao qual denominaram de Trypanosoma freitasi n.sp. Vale assinalar, as baixíssimas parasitemias observadas.

A seguir Daene ${ }^{1}$ (1964), refere o achado de um $D$. marsupialis, capturado nas matas do Ltinga, arredores de Belém do Pará, simultaneamente infectado pelo $T$. crusi e pelo $T$. freitasi.

Foi através desses achados que Hoare ${ }^{3}$ (1972) considerou válida a espécie. classificando-a como Trypanosoma (Mega- trypanum) freitasi Rêgo, Magalhães e Siqueira, 1957.

Consulta bibliográfica, na verdade incompleta, não revelou achados recentes, sendo pois considerado de interesse o reencontro do $T$. (M.) freitasi, no Estado de São Paulo. Brasil. Confirmam-se assim os achados anteriores do parasito, bem como, abrem-se perspectivas para o estudo do seu ciclo de vida e outros.

\section{MATERIAL E METODOS}

As equipes da SLCEN, dando prosseguimento às observações em andamento relativas aos hábitos e comportamento do Panstrongylus megistus, não só procuram triatomíneos nas casas e prédios anexos, como se esforçam por capturar os animais

* Da Superintendência de Controle de Endemias - SUCEN — Secretaria da Saúde - Rua Tamandaré, 649 - São Paulo, SP - Brasil e do Departamento de Epidemiologia da Faculdade de Saúde Pública da USP - Av. Dr. Arnaldo, 715 - São Paulo, SP - Brasil.

* Do Departamento de Epidemiologia da Faculdade de Saúde Pública da USP - Av. Dr. Arnaldo, 715 - São Paulo, SP - Brasil.

**: Da Superintendência de Controle de Endemias - SUCEN - Secretaria da Saúde - Rua Tamandaré, 649 - São Paulo, SP - Brasil. 
ROCHA E SILVA, E. O. et al. - Novo encontro do Trypanosoma

(Megatrypanum) freitasi, parasita do gambá. Rev. Saúde públ., S. Paulo, 10:121-4, 1976.

sinantrópicos ocasionalmente encontrados nesses locais. Esses vertebrados, freqüentemente marsupiais do gênero Didelphis, encaminhados ao laboratório de MojiGuaçu, são examinados visando o encontro e. quando possível. o isolamento do $T$. cruzi.

Recentemente (abril/75). um exemplar do $D$. azarae capturado num paiol. localizado no Bairro do Bréjinho Imunicípio de S. J. do Rio Pardol. mostrou ao exame à fresco a presença. no sangue. de flagelado com dimensões e movimento incomuns. A coloração pelo Giemsa. das gotas de sangue coletado da cauda e por punção intracardiaca. evidenciou raras e belas formas de um tripanossomo. Posteriormente observado também em outros exemplares, procedentes da mesma localidade.

Além do estudo morfológico, tentou-se o cultivo em NNX. o desenvolvimento no iubo intestinal do $P$. megistus e $T$. infestans e seu isolamento. através de inoculação em animais de laboratório.

\section{RESULTA DOS}

\subsection{Morfologia}

As Figuras 1. 2 e 3 com legendas que resumem a descrição original de Rêgo et al. ${ }^{2}$. dizem bem da identificação como Trupanosoma (Megatrypanum) freitasi.

As lâminas sintípicas da espécie. depositadas no Departamento de Parasitologia. da Faculdade de Medicina de Ribeirão Preto. tendo em vista o tempo decorrido. não foram encontradas para comparação.

Após o achado inicial. retornou-se ao Bairro Bréjinho visando a captura de outros animais. resultando: 29 exemplares do $D$. azarae. 2 roedores (Oryzomys) e 3 morcegos (Phylostomidae).

Apenas entre Didelphis conseguiu-se detectar tripanossomos. sendo que, quatro $(13.79 \%)$ apresentaram escasso parasitismo pelo $T$. (M.) freitasi e oito $(27.59 \%)$, pelo $T$. (S.) cruzi.

Lm desses exemplares. como já relatara Daene ${ }^{1}$, também apresentou infecção dupla.

\subsection{Xenodiagnóstico}

Os marsupiais e roedores, foram submetidos a xenodiagnósticos, realizados com exemplares do $T$. infestans e $P$. megistus.

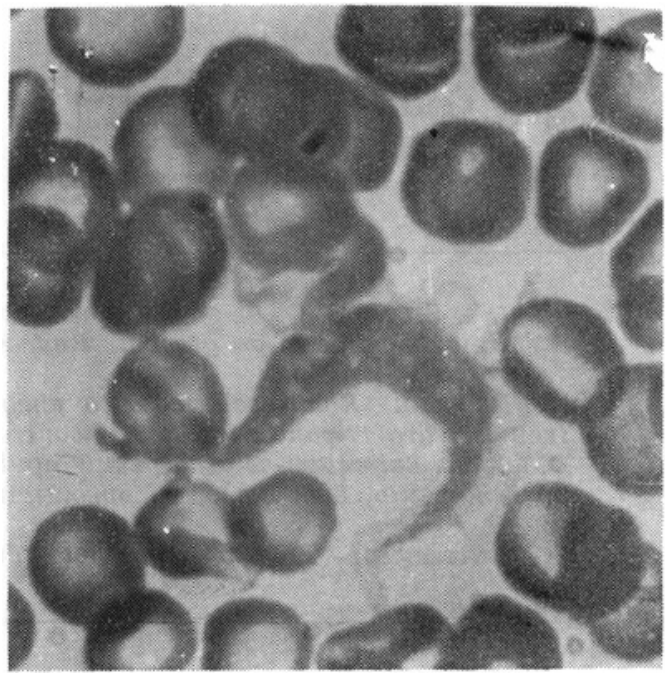

Fig. 1 - T. (Mr) freitasi: Corpo largo, principalmente na região mediana, extremidades afiladas. No citoplasma, diferentes intensidades de coloração e pequenos vacúolos.

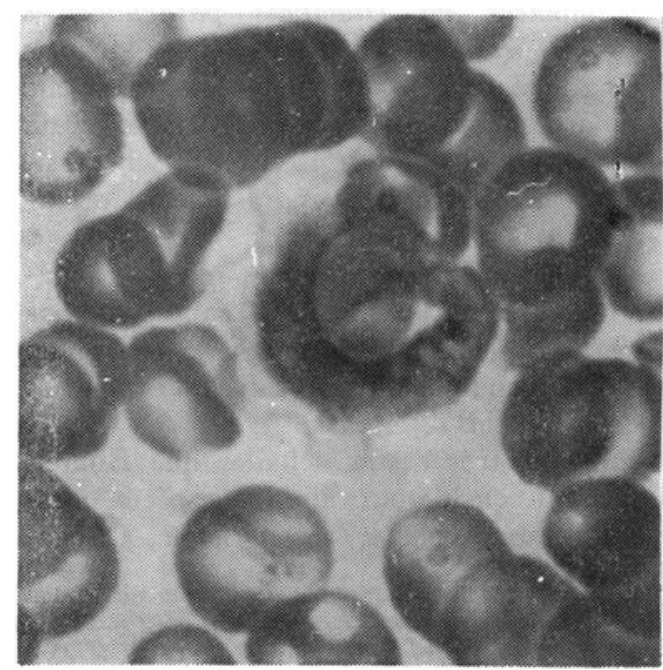

Fig. 2 - T. (M.) freitasi: Forma geral em C. Cinetoplasto marginal, afastado da extremidade posterior. 
ROCHA E SILVA, E. O. et al. - Novo encontro do Trypanosoma (Megatrypanum) freitasi, parasita do gambá. Rev. Saúde públ., S. Paulo, 10:121-4, 1976.

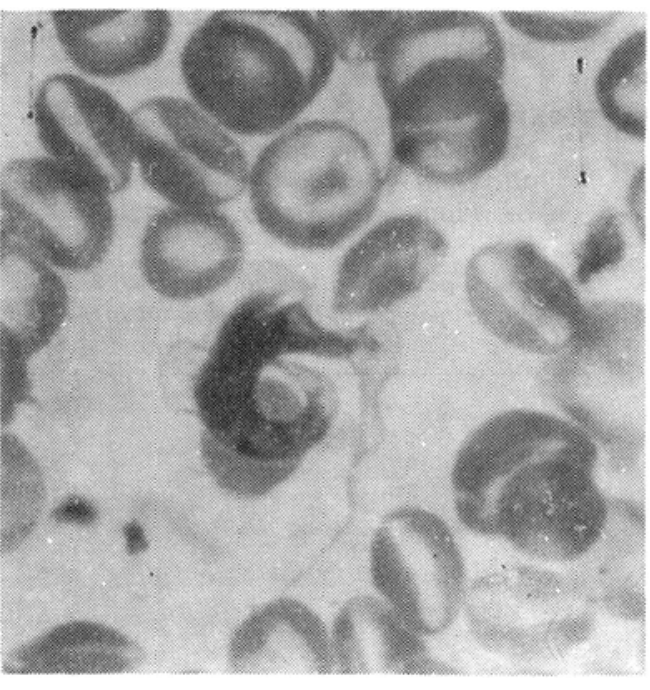

Fig. $3-T$. $\left(M_{\text {. }}\right)$ freitasi: Membrana ondulante apresentando ondulaçōes eviđentes. Nücleo ovoldal, pouco evidente.

Este último, ressalte-se, freqüentemente encontrado na área, em ninhos de gambás.

Os xenos realizados, positivaram apenas para $T$. cruzi.

\subsection{Inoculação}

O sangue dos animais parasitados pelo $T$. (M.) freitasi, inoculado por via intraperitoneal em animais de laboratório: camundongo, rato branco e hamster, não causou infecção. Alguns inoculados com $T$. cruzi, isolado pelos xenos, se infectaram apresentando no sangue formas características da espécie.

\subsection{Cultivo}

Todas as tentativas de cultivo, em meio NNN, resultaram negativas. Embora seja possivel questionar a validade desses resultados, tendo em vista a escassa e irregular presença de parasitos nas fontes disponíveis.

\section{CON CLUSOES}

4.1. No Bairro do Bréjinho, proximidades de S. J. do Rio Pardo, foram detectadas duas espécies de tripanosomatídeos, parasitando, isolada ou concomitantemente, o Didelphis azarae.

A prevalência maior foi do $T$. cruzi, observado também na área infectando o $P$. megistus.

O $T$. freitasi, foi encontrado com prevalência menor e observado somente nos dedelfídeos. Apresentou sempre parasitemia escassa e irregular.

Todas observações indicam que a transmissão se processa sem passagem pelos triatomíneos, como já fora observado.

4.2. Quanto ao valor prático deste achado, cite-se Rêgo et al. ${ }^{2}$ que referiram a possibilidade de confusão com o $T$. cruzi, nos esfregaços e gotas grossas à fresco, se observada apenas a movimentação das hemácias pelo flagelado. A dúvida desaparecerá com a visualização do parasito.

De qualquer maneira, recomenda-se a adequada fixação e coloração do material positivo.

\section{RSPU-B/305}

Rocha E Silva, E. O. et al. - [A new finding of Trypanosoma (Megatrypanum) freitasi, parasite of the opossum]. Rev. Saúde públ., S. Paulo, 10:121-4, 1976.

Summary: $A$ new finding of the Trypanosoma (Megatrypanum) freitasi a rare trypanosome encountered in the blood of marsupial's of the genus Didelphis, is reported. The feeble and irregular parasitemy, the difficulties for its isolation, as will as the concomitant observation of Trypanosoma cruzi, is mentioned.

UNITERMS: Trypanosomes. Trypanosomiasis, South American Reservoirs. 
ROCHA E SILVA, E. O. et al. - Novo encontro do Trypanosoma (Megatrypanum) freitasi, parasita do gambá. Rev. Saúde públ., S. Paulo, 10:121-4, 1976.

\section{REFERENCIAS BIBLIOGRAFICAS}

1. DAENE, L. M. - Tripanosomatídeos de Mamíferos da Região Amazônica. III. Hemoscopia e xenodiagnóstico de animais silvestres dos arredores de Belém, Pará. Rev. Inst. Med. trop., S. Paulo, 6:225-32, 1964.

2. REGO, S. F. N. et al - Lm novo tripanossomo do gambá. Trypanosoma frei- tasi, N. SP. Rev. bras. Malar., 9: $277-84,1957$.

3. HOARE, C. A. - Megatrypanum from Marsupials. In: The Trypanosomes of Mammals. Oxford, Blackwell Scientific Publications, 1972. p. 173-4

Recebido para publicaşao em $15 / 12 / 1975$ Aprovado para publicação em 05/01/1976 